\title{
The high-mass stellar IMF in different environments
}

\author{
Pavel Kroupa \\ Argelander Institute for Astronomy, University of Bonn, \\ Auf dem Hügel 71, D-53121 Bonn, Germany \\ email: pavel@astro.uni-bonn.de
}

\begin{abstract}
The massive-star IMF is found to be invariable. However, integrated IMFs probably
\end{abstract} depend on galactic mass.

Keywords. stars: luminosity function, mass function; stars: pre-main-sequence

To set the stage it is useful to emphasis that the Salpeter (1955) mass function (MF), $\xi(m)=k m^{-\alpha}, \alpha=2.35$, is strictly valid only for stellar masses with $0.4 \lesssim m / \mathrm{M}_{\odot} \lesssim 10$. The number of stars in the mass interval $m, m+d m$ is $d N=\xi(m) d m$. Given statistical noise in the IMF of otherwise equal systems the question we need to answer is whether there is any significant empirical evidence for systematic variation of the IMF with the physical conditions of star formation. This question is of fundamental importance for star-formation theory because an observed systematic variation poses constraints on the theory. The question is also of fundamental importance for cosmology because the physical conditions of star formation have changed dramatically over a cosmological epoch implying possible systematic changes of the young stellar populations with cosmic time and therefore systematic changes in the properties of galaxies. Very different theoretical approaches lead to one common result, namely that the average stellar mass ought to shift towards larger values with decreasing metallicity. Thus, Adams \& Fatuzzo (1996) develop a model of the origin of the IMF based on the notion that stars regulate their own masses through feedback, while Larson (1998) investigates the systematic changes of the IMF as a result of the temperature-dependence of the Jeans mass. Recent developments based on a change of the equation of state as a result of dust processes may be an avenue of explaining the general absence of a variation of the stellar mass at which the IMF peaks (Bonnell et al. 2006). Observationally, the IMF has been constrained above a few $\mathrm{M}_{\odot}$ by Massey (2003) for populations in the Milky Way, the Large and Small Magellanic Clouds and for different densities. The massive-star IMF has been found to have an invariable "Salpeter/Massey" index $\alpha=2.3 \pm 0.2$. This insensitivity to the physical conditions needs to be understood, and perhaps suggests that massive star formation is dominated by scale-free processes such as coagulation and/or competitive accretion in the very dense environments where massive stars form (Bonnell et al. 2006). For entire galaxies the composite IMF probably depends on galaxy type such that low-mass galaxies have very steep indices (Weidner \& Kroupa 2006), despite the invariability of the IMF.

\section{References}

Adams, F. C., \& Fatuzzo, M. 1996, ApJ, 464, 256

Bonnell, I. A., Larson, R. B., \& Zinnecker, H. 2006, in: B. Reipurth, D. Jewitt \& K. Keil (eds.), Protostars and Planets $V$ (Tucson: Univ. Arizona Press), p. 951

Weidner, C., \& Kroupa, P. 2006, MNRAS, 365, 1333

Larson, R. B. 1998, MNRAS, 301, 569

Massey, P. 2003, ARAA, 41, 15

Salpeter, E. E. 1955, ApJ, 121, 161 